\title{
Diagnostic tests for SARS-CoV-2: current status and issues
}

\author{
Sraboni Mazumder ${ }^{1}{ }^{1}$, Md Monirul Hoque $^{2}$ \\ ${ }^{1}$ Department of Microbiology, Ibrahim Medical College, Shegun Bagicha, Dhaka, Bangladesh, ${ }^{2}$ Department \\ of Pathobiology, College of Veterinary Medicine, Auburn University, Alabama, USA
}

\begin{abstract}
The current coronavirus disease 2019 (COVID-19) pandemic has affected the whole world. Accurate, rapid and affordable diagnostic testing for COVID-19 is crucial to prevent and control this global pandemic. This paper reviews the current status and issues related to diagnostic tests for COVID-19.
\end{abstract}

IMC J Med Sci 2020; 14(2): 004. EPub date: 24 October 2020

\section{Introduction}

The current outbreak of coronavirus disease 2019 (COVID-19) which emerged in Wuhan, China, is caused by a novel coronavirus named severe acute respiratory syndrome coronavirus 2 (SARS-CoV-2) [1]. World Health Organization (WHO) declared SARS-CoV-2 a pandemic on March 11, 2020 [2]. Accurate, rapid and affordable diagnostic testing for COVID-19 is crucial to prevent and control this global pandemic. The global approach to SARS-CoV2 testing has been non-uniform. In South Korea, individuals with respiratory illness and any contacts with COVID-19 are tested whereas Spain initially limited testing to individuals with severe symptoms or those at high risk of developing them [3].

The vital role of highly sensitive and specific diagnostic assay in the control of infectious epidemic was evidenced around two decades back, in 2002/2003, when SARS-CoV emerged in Southeast Asia and when MERS-CoV emerged in Middle East back in 2012. The concerted efforts of public health authorities by means of rapid testing of suspected cases interrupted the chain of transmission and helped contain the outbreak. Moreover, valid, rapid, sensitive and specific laboratory diagnostic tools are essential for proper case identification, timely management of the patients, contact tracing, animal source finding, and rationalization of infection control measures in COVID-19 [4].
Several approaches have been used to devise rapid and affordable test(s) to detect COVID-19 cases efficiently and as early as possible to prevent and control this highly transmissible disease. The tests include virus culture, molecular technique for detection of viral nucleic acid and immunoassays.

\section{Virus culture}

Diagnosis using viral culture is not useful, as it takes at least 3-6 days for SARS-CoV-2 to cause apparent cytopathic effects in selected cell lines, such as VeroE6 cells. Moreover, isolation of the virus requires highly skilled manpower; expensive equipment and biosafety level 3 facilities, which are not available in most health care institutions [5]

\section{Molecular assays}

A real-time RT-PCR (reverse transcriptase polymerase chain reaction) method is recommended for detecting SARS-CoV-2 during the period of viral shedding in acute phase of COVID19, till date. However, this method, when used alone, has limitation in the detection of the virus during different phases of the illness as the viral load of SARS-CoV-2 is very high when symptoms appear (overall >1 $>10^{6}$ copies $/ \mathrm{mL}$ ) among different clinical specimens [6] and declines 
steadily $[7,8]$. Another pressing issue regarding using RT-PCR to detect COVID-19 case is the falsenegative and false-positive results. Wang et al. reported the failure to diagnose many suspected cases having conventional clinical COVID-19 features including specific computed tomography (CT) images led to inefficient separation of many potential cases and hindered the control strategy [9]. Following infection, SARS-CoV-2 undergoes immunologic pressure in humans and accumulate mutations, which may affect not only its transmissibility and virulence but also its detectability with the same RT-PCR kit overtime [10]. One study detected 93 mutations among 86 complete or near complete genome of SARS-CoV-2 [11]. Similarly, mutations in the primer and probe target regions of the SARS-CoV-2 genome for RTPCR may produce false-negative results. Although, in order to mitigate this problem, several types of SARS-CoV-2 RT-qPCR kit have been devised targeting the conserved regions of the viral genome and targeting multiple target gene amplification. However, variability resulting in mismatches between the primers and probes and the target sequences might reduce the assay performance. Moreover, the viral load of SARS-CoV-2 in different anatomic sites, sampling timing, sampling procedures and stage of the disease play important roles in producing false-negative results [12].

Specimens are generally collected from both the upper respiratory tract (URT; nasopharynx and oropharynx) and lower respiratory tract (LRT; expectorated sputum, endotracheal aspirate, or bronchoalveolar lavage) for COVID-19 testing by RT-PCR. The virus is also detected in fecal and blood specimens [13]. The sensitivity/positivity rate of RT-PCR in various biological samples of COVID-19 patients is shown in Table-1. The sensitivity/ positivity rate varies from $3.03 \%$ to $93 \%$ in various clinical samples. A study observed strong correlation of viraemia with the disease severity [14]. SARS-CoV-2 quantification in plasma/serum could also represent a potentially useful early diagnostic and prognostic tool [15]. According to Guangzhou CDC, virus can be detected in upper respiratory samples 1-2 days prior to symptom onset and persists for 7-12 days in moderate cases and up to 2 weeks in severe cases [13]. Prolonged viral shedding from nasopharyngeal aspirates - up to at least 24 days after symptom onset - was reported among COVID-19 patients in Singapore [16]. Viral RNA has been detected in feces in up to $30 \%$ of patients from day 5 following onset of symptoms and has been noted for up to 4-5 weeks in moderate cases [13]. Fang et al. found first RTPCR test positive in $71 \%$ cases after studied on first throat swab or sputum samples from 51 patients. In second RT-PCR, another $23 \%$ cases became positive who were initially negative. In third and fourth RT-PCR, another $4 \%$ and $2 \%$ cases became positive [17]. Another study conducting serial RTPCR testing showed the mean time from an initial negative RT-PCR to subsequent positive RT-PCR was 5.1 days ( \pm 1.5 days) [18]. Regarding asymptomatic patients, Arons et al. reported that more than half of subjects with positive test results were asymptomatic at the time of testing [19]. Zou et al. reported that the viral load of asymptomatic patients was similar to symptomatic patients, indicating a transmission potential of asymptomatic or pre-symptomatic patients. The study reported that patients with few or no symptoms had modest levels of detectable viral RNA in the oropharynx for at least 5 days [20].

Table-1: The sensitivity/positivity rate of $R T-P C R$ in different specimens during acute phase of COVID19 patients

\begin{tabular}{lcc}
\hline \multicolumn{1}{c}{ Specimen } & $\begin{array}{c}\text { Sensitivity/ } \\
\text { positivity } \\
\text { rate (\%) }\end{array}$ & References \\
\hline Nasopharyngeal swab & $63-89$ & {$[21,22]$} \\
Oropharyngeal swab & $32-84$ & {$[21,23]$} \\
Brochoalveolar lavage & 93 & {$[21]$} \\
Sputum & 72 & {$[21]$} \\
Blood & $3.03-15$ & {$[24,25]$} \\
Feces & 9.83 & {$[24]$} \\
Anal swab & $10-21.2$ & {$[24,26]$} \\
\hline
\end{tabular}

RT-PCR assay targets the open reading frames (ORF1a and ORF1b), non-structural protein (nsp14), RNA-dependent RNA polymerase (RdRp), envelope glycoproteins spike (S), envelope (E), nucleocapsid $(\mathrm{N})$, or helicase (Hel) gene of SARS-CoV-2. To avoid potential cross-reaction with other endemic coronaviruses as well as potential genetic drift of 
SARS-CoV-2, at least two molecular targets should be included in the assay. Various investigators in different countries have used a number of these molecular targets for real-time RT-PCR assays [27]. In the United States, the US Centers for Disease Control and Prevention (CDC) recommends two nucleocapsid protein targets (N1 and N2) [28] while WHO recommends first line screening with the $E$ gene assay followed by a confirmatory assay using the RdRp gene [29]. Another study in Hong Kong, China used two targets for their RT-PCR assay; the first used the nucleocapsid for screening followed by confirmation by the open reading frame $1 b$ [30]. Chan et al. developed and compared the performance of three novel real-time RT-PCR assays targeting the RdRp/Hel, $\mathrm{S}$, and $\mathrm{N}$ genes of SARS-CoV-2. Among them, the COVID-19-RdRp/Hel assay had the lowest limit of detection in vitro and higher sensitivity and specificity [31]. The analytical sensitivity of different RT-PCR test kits varies from 0.15 to $100 \mathrm{copy} / \mu \mathrm{L}$ [32].The US CDC recommends that negative results of real time RT-PCR testing for SARS-CoV-2 from at least two sequential respiratory tract specimens collected at least 24 hours apart can be considered to discontinue transmission-based precautions [33].

\section{Immunoassays}

Success of PCR-based diagnostics relies on timing and technique of sampling, stage of the infection, type of sample, the kinetics of viraemia and shedding of virus throughout the course of infection. Moreover, inadequate access to reagents, expensive equipment and bio-safety facilities have resulted in low efficiency in handling large number of samples in-time delivery of reports. Therefore, serological testing is crucial to complement the RT-qPCR. In addition, serology is used as an important tool to monitor the evolution of an outbreak, retrospective studies of asymptomatic and mild cases and animal reservoir identification [34,35]. However, devising serologic assays targeting immunogenic proteins is difficult because closely related viruses may share common epitopes that elicit cross-reactive and crossneutralizing antibodies. Within a genus, antibodies against other coronaviruses might cross-react and such cross-reactive conserved viral proteins limit the use of whole virus-based assays, for example, immunofluorescence assay (IFA) [7]. Also whole virus based assays primarily require viral culture which is difficult to establish.

Several immunoassays have been developed for rapid detection of SARS-CoV-2 antigens or antibodies to overcome this hurdle. Immunoassays tests include rapid lateral flow assays, ELISA and chemiluminescence. These serological tests provide the advantage of fast and low-cost detection of SARS-CoV-2 but are likely to suffer from poor sensitivity during acute phase of the disease [27].

According to recent studies, serological testing identifies convalescent cases or people with milder disease or patients who present late with a very low viral load, below the detection limit of RT-PCR assays. One study evaluated two recombinant SARS-CoV-2 nucleocapsid protein ( $\mathrm{rN}$ ) and spike protein ( $r S$ ) based ELISA kits for detection of IgM and IgG antibodies. They found high sensitivity in samples collected from patients 10 days postdisease onset. They observed that IgM and IgG positivity rate increased with increasing interval of days following onset of disease [1]. Serum IgG was found to rise at the same time or earlier than those of IgM against SARS-CoV-2. It was reported that a higher proportion of patients had earlier IgG than IgM seroconversion probably due to lower sensitivity of the IgM ELISA [8]. CDC's serologic test designed to detect antibodies against SARS-CoV-2 spike protein antigen has a specificity of greater than $99 \%$ and a sensitivity of $96 \%$. It can be used to identify past SARS-CoV-2 infection in people who were infected at least 1 to 3 weeks previously [36]. Different antibody detection assays have been approved in different countries for diagnostic and/or research use. The sensitivity and specificity of those methods are shown in Table-2. Several antigens mainly spike and nucleocapsid proteins have been used as capture-antigen in ELISA and lateral flow assay/rapid diagnostic test (RDT) to diagnose IgG and/or IgM against SARS-CoV-2 [37]. Nevertheless, cross-reactivity of antibodies to closely related viruses is a potential issue to interpret the serological test results.

A South Korean antigen detection kit reported $84.38 \%$ and $100 \%$ sensitivity and specificity respectively using nasopharyngeal swabs from 202 
symptomatic patients for detection of SARS-CoV-2 antigen [38]. Quidel Sofia, USA SARS antigen test kit has been reported to detect antigen targeting nucleocapsid protein from SARS-CoV-2 with $96.7 \%$ sensitivity within five days of the onset of symptoms. The kit detects viral antigen in nasopharyngeal or nasal swab using immunofluorescence-based lateral flow technology [39]. Another antigen detection kit developed in Japan that detects neucleocapsid protein antigen of SARS-CoV-2 in nasopharyngeal swab using immunochromatographic assay has almost similar sensitivity and specificity [40].

Table-2: Sensitivity and specificity of antibody detection assays for COVID-19 [37]

\begin{tabular}{llll}
\hline $\begin{array}{l}\text { Test } \\
\text { format }\end{array}$ & Antibody & $\begin{array}{c}\text { Sensitivity } \\
\mathbf{( \% )}\end{array}$ & $\begin{array}{c}\text { Specificity } \\
\mathbf{( \% )}\end{array}$ \\
\hline ELISA & IgG & $>90-100$ & $>95-100$ \\
& IgM & $>95$ & $>95$ \\
RDT & IgG and IgM & $82-93.8$ & $95.6-100$
\end{tabular}

Note: ELISA=enzyme linked immunosorbent assay; $R D T=$ rapid diagnostic assay.

Transformation of the script of laboratory based diagnostic approach into a self-conducted, noninvasive, rapid, convenient, cheap and available over-the-counter diagnostic test, be it less sensitive than the current RT-PCR or serology assay, would enable mass people detect themselves as suspected cases. Thus they could self-isolate far ahead of time than if they were to be diagnosed by the conventional laboratory tests. As long as these people stay home, it would provide a kind of artificial herd immunity which will interrupt the chain of transmission to impede the pandemic. Moreover, these suspected cases would be able to confirm the infection status later by the more specific laboratory tests. The most important issue is that, this strategy would best utilize the minimal resources and the narrow window of time that is not achievable with more sensitive but expensive and time consuming PCR tests. Sherlock Biosciences of Harvard's Wyss Institute for Biologically Inspired Engineering and E25Biorooting from Massachusetts Institute of Technology (MIT) and Harvard have developed an inexpensive paper-based test which can be conducted at home with saliva or nasal mucous, just like doing at-home pregnancy test [41].

\section{Symptom-based diagnosis}

A simple and technology independent diagnostic tool, if available, would be immensely valuable to handle the present COVID-19 pandemic. Historically, after initial detection and confirmation of the offending microbe, many previous epidemics were combated without laboratory testing of every case. Subsequently, the cases were detected and managed by typical clinical features of the disease. Therefore, clinical symptom and sign based diagnostic approach may also be a valuable and useful instrument to diagnose COVID-19 in places where RT-PCR or other serological tests are not easily available. There is paucity of studies with regard to the sensitivity and specificity of such symptom based diagnosis of COVID-19. Few studies that considered symptoms based diagnosis of COVID19 assessed symptoms alone. A Cochrane systematic review reported that till the end of April 2020 no study assessed combinations of different signs and symptoms to diagnose a case of COVID-19. The review revealed a sensitivity of $50 \%$ and specificity of $90 \%$ when six symptoms (cough, sore throat, fever, myalgia or arthralgia, fatigue, and headache) were considered [42]. The symptom of "sudden smell loss" has been associated with $97 \%$ specificity and a sensitivity of $65 \%$ with positive and negative predictive values of $63 \%$ and $97 \%$ respectively for COVID-19 [43].

Therefore, combined symptoms and signs (may be including imaging characteristics) based diagnostic tool for COVID-19 should be developed and tested for sensitivity and specificity in different areas and healthcare settings. Such technology independent tool might help in primary care, emergency or in telemedicine services in resource poor countries/regions to identify COVID-19 patients at low cost and thus would minimize its spread. Moreover, it would reduce the cost of diagnosing COVID-19 even in hospitals with all facilities by avoiding expensive RT-PCR test in every case. 


\section{Conclusion}

The pandemic of SARS-CoV-2 infection has emphasized the importance of simple, fast and affordable high quality diagnostic tools to limit the spread as well as to appropriately treat the COVID-19 patients. Further studies are needed to develop easy to use assays of similar sensitivity and specificity of RT-PCR. Symptom/sign based technology independent diagnostic tool for detection of COVID19 deserves further attention because that can be used at all levels of healthcare facilities.

\section{Acknowledgement}

We acknowledge the idea and advice of Prof. J. Ashraful Haq, Department of Microbiology, Ibrahim Medical College, Dhaka, Bangladesh.

\section{References}

1. Liu W, Liu L, Kou G, Zheng Y, Ding Y, Ni W, et al. Evaluation of nucleocapsid and spike proteinbased ELISAs for detecting antibodies against SARS-CoV-2. J Clin Microbiol. 2020; 58(6): e00461-20.

2. World Health Organization. Director-General's opening remarks at the media briefing on COVID-19 - 11 March 2020. Geneva: World Health Organization; 2020.

3. Black JRM, Bailey C, Swanton C. COVID-19: the case for health-care worker screening to prevent hospital transmission. Lancet. 2020; 395(10234): 1418-1420.

4. Meyer B, Drosten C, Müller MA. Serological assays for emerging coronaviruses: challenges and pitfalls. Virus Res. 2014; 194: 175-183.

5. Chan JF, Yip CC, To KK, Tang TH, Wong SC, Leung $\mathrm{KH}$, et al. Improved molecular diagnosis of COVID-19 by the novel, highly sensitive and specific COVID-19-RdRp/Hel real-time reverse transcription-PCR assay validated in vitro and with clinical specimens. J Clin Microbiol. 2020; 58(5).

6. Pan $Y$, Zhang D, Yang P, Poon LLM, Wang Q. Viral load of SARS-CoV-2 in clinical samples. Lancet Infect Dis. 2020; 20(4): 411-412.
7. Yong SEF, Anderson DE, Wei WE, Pang J, Chia WN, Tan CW, et al. Connecting clusters of COVID-19: an epidemiological and serological investigation. Lancet Infect Dis. 2020; 20(7): 809-815.

8. To KK, Tsang OT, Leung WS, Tam AR, Wu TC, Lung DC, et al. Temporal profiles of viral load in posterior oropharyngeal saliva samples and serum antibody responses during infection by SARS-CoV-2: an observational cohort study. Lancet Infect Dis. 2020; 20(5): 565-574.

9. Wang $Y$, Kang $H$, Liu $X$, Tong Z. Combination of RT-QPCR testing and clinical features for diagnosis of COVID-19 facilitates management of SARS-CoV-2 outbreak. J Med Virol. 2020; 92(6): 538-539.

10. Shen Z, Xiao Y, Kang L, Ma W, Shi L, Zhang L, et al. Genomic diversity of severe acute respiratory syndrome-coronavirus 2 in patients with coronavirus disease 2019. Clin Infect Dis. 2020; 71(15): 713-720.

11. Tung $P$. Genetic diversity and evolution of SARS-CoV-2. Infect Genet Evol. 2020; 81: 104260.

12. Alireza T, Ardebili A. Real-Time RT-PCR in COVID-19 detection: issues affecting the results. Expert Rev Mol Diagn. 2020; 20(5): 453-454.

13. World Health Organization. Report of the WHO-China joint mission on coronavirus disease 2019 (COVID-19). Geneva: World Health Organization; 2020.40 p.

14. Chen $W$, Lan $Y$, Yuan $X$, Deng $X$, Li Y, Cai X, et al. Detectable 2019-nCoV viral RNA in blood is a strong indicator for the further clinical severity. Emerg Microbes Infect. 2020; 9(1): 469-473.

15. Yan $Y$, Chang L, Wang L. Laboratory testing of SARS-CoV, MERS-CoV, and SARS-CoV-2 (2019nCoV): current status, challenges, and countermeasures. Rev Med Virol. 2020; 30(3): e2106.

16. Young BE, Ong SWX, Kalimuddin S, Low JG, Tan SY, Loh J, et al. Epidemiologic features and clinical course of patients infected with SARSCoV-2 in Singapore. JAMA. 2020; 323(15): 1488-1494. 
17. Fang $Y$, Zhang $H$, Xie J, Lin $M$, Ying $L$, Pang $P$, et al. Sensitivity of chest CT for COVID-19: comparison to RT-PCR. Radiology. 2020; 296(2): E115-E117.

18. Ai T, Yang Z, Hou H, Zhan C, Chen C, Lv W, et al. Correlation of chest CT and RT-PCR testing for coronavirus disease 2019 (COVID-19) in China: A Report of 1014 Cases. Radiology. 2020; 296(2): E32-E40.

19. Arons MM, Hatfield KM, Reddy SC, Kimball A, James $A$, Jacobs JR, et al. Presymptomatic SARS-CoV-2 infections and transmission in a skilled nursing facility. N Engl J Med. 2020; 382(22): 2081-2090.

20. Zou L, Ruan F, Huang $M$, Liang L, Huang $H$, Hong Z, et al. SARS-CoV-2 viral load in upper respiratory specimens of infected patients. $N$ Engl J Med. 2020; 382(12): 1177-1179.

21. Wang W, Xu Y, Gao R, Lu R, Han K, Wu G, et al. Detection of SARS-CoV-2 in different types of clinical specimens. JAMA. 2020; 323(18): 18431844.

22. Jamal AJ, Mozafarihashjin $M$, Coomes $E$, Powis J, Li AX, Paterson A, et al. Sensitivity of nasopharyngeal swabs and saliva for the detection of severe acute respiratory syndrome coronavirus 2 (SARS-CoV-2). Clin Infect Dis. 2020; 25: ciaa848.

23. Centers for Disease Control and Prevention. Performance of oropharyngeal swab testing compared with nasopharyngeal swab testing for diagnosis of coronavirus disease 2019-United States, January 2020-February 2020. United States: Oxford University Press for the Infectious Diseases Society of America; 2020. 4 p.

24. Wu J, Liu J, Li S, Peng Z, Xiao Z, Wang X, et al. Detection and analysis of nucleic acid in various biological samples of COVID-19 patients. Travel Med Infect Dis. 2020; 18: 101673.

25. Chang L, Yan Y, Wang L. Coronavirus disease 2019: coronaviruses and blood safety. Transfus Med Rev. 2020; 34(2): 75-80.

26. Lin $W$, Xie $Z$, Li $Y$, Li L, Wen $C$, Cao $Y$, et al. Association between detectable SARS-COV-2 RNA in anal swabs and disease severity in patients with coronavirus disease 2019. J Med Virol. 2020; 10.

27. Tang YW, Schmitz JE, Persing DH, Stratton CW. The laboratory diagnosis of COVID-19 infection: current issues and challenges. J Clin Microbiol. 2020; 58(6): e00512-20.

28. Holshue $\mathrm{ML}$, DeBolt $\mathrm{C}$, Lindquist $\mathrm{S}$, Lofy $\mathrm{KH}$ Wiesman J, Bruce H, et al. First case of 2019 novel coronavirus in the United States. N Engl J Med. 2020; 382: 929-936.

29. Corman VM, Landt $O$, Kaiser M, Molenkamp R, Meijer A, Chu DKW, et al. Detection of 2019 novel coronavirus (2019-nCoV) by real-time RT-PCR. Euro Surveill. 2020; 25(3).

30. Chan JF, Yuan S, Kok KH, To KK, Chu H, Yang J, et al. A familial cluster of pneumonia associated with the 2019 novel coronavirus indicating person-to-person transmission: a study of a family cluster. Lancet. 2020; 395: 514-523.

31. Chan JF, Yip CC, To KK, Tang TH, Wong SC, Leung $\mathrm{KH}$, et al. Improved molecular diagnosis of COVID-19 by the novel, highly sensitive and specific COVID-19-RdRp/Hel real-time reverse transcription-polymerase chain reaction assay validated in vitro and with clinical specimens. $J$ Clin Microbiol. 2020; 4: 310-320.

32. Giri B, Pandey S, Shrestha R, Pokharel K, Ligler FS, Neupane BB. Review of analytical performance of COVID-19 detection methods. Anal Bioanal Chem. 2020; 18: 1-14.

33. Centers for Disease Control and Prevention. Interim guidance for discontinuation of transmission-based precautions and disposition of hospitalized patients with COVID-19. United States of America: Centers for Disease Control and Prevention; 2020.

34. Chen W, Xu Z, Mu J, Yang L, Gan H, Mu F, et al. Antibody response and viraemia during the course of severe acute respiratory syndrome (SARS)-associated coronavirus infection. J Med Microbiol. 2004; 53(Pt 5): 435-438.

35. Guan $Y$, Zheng BJ, He YQ, Liu XL, Zhuang ZX, Cheung $\mathrm{CL}$, et al. Isolation and characterization of viruses related to the SARS coronavirus from 
animals in Southern China. Science. 2003; 10 302(5643): 276-278.

36. Centers for Disease Control and Prevention. Serology testing for COVID-19. United States of America: Centers for Disease Control and Prevention; 2020.

37. Johns Hopkins Bloomberg School of Public Health. Serology-based tests for COVID-19: United States: Johns Hopkins University; 2020.

38. SD BIOSENSOR. Standard Q COVID-19 Ag. Korea: SD BIOSENSOR; 2020.

39. Quidel. Sofia SARS Antigen FIA. USA: Quidel; 2020.

40. Fujirebio. ESPLINE ${ }^{\circledast}$ SARS-CoV-2. Japan: Fujirebio; 2020.
41. Powell A. Cheap, frequent COVID tests could be 'akin to vaccine,' professor says. The Harvard Gazette. Harvard University; 2020.

42. Struyf $T$, Deeks JJ, Dinnes J, Takwoingi $Y$, Davenport $C$, Leeflang MMG, et al. Signs and symptoms to determine if a patient presenting in primary care or hospital outpatient settings has COVID-19 disease. Cochrane Database Syst Rev. 2020; 7(7): CD013665.

43. Haehner A, Draf J, Dräger $S$, de With $K$, Hummel T. Predictive value of sudden olfactory loss in the diagnosis of COVID-19. ORL $J$ Otorhinolaryngol Relat Spec. 2020; 82(4): 175180. 